\title{
STUDY ON THE SPATIOTEMPORAL FEATURES AND EVOLUTION OF ALPINE NOMADIC SETTLEMENTS FROM THE PERSPECTIVE OF ECOLOGICAL WISDOM: CASE STUDY OF QIONGKUSHITAI VILLAGE IN XINJIANG, CHINA
}

\author{
MENG, F. L. ${ }^{1,2}-\mathrm{HE}, \mathrm{Y}^{2 *}$ \\ ${ }^{1}$ Shihezi University, School of Literature and Art, Shihe-zi 832003, Xinjiang, China \\ No. 225, No. 31 Beishi Road, Shihezi City, Xinjiang, China \\ (e-mail: mengfuli2008@163.com; phone: +86-180-9598-3089) \\ ${ }^{2}$ HuaZhong University of Science And Technology, School of Architecture and Urban Planning \\ Wuhan 430079, Hubei, China \\ ${ }^{*}$ Corresponding author \\ e-mail: 330337342@qq.com; phone:+86-136-0713-9805 \\ (Received $24^{\text {th }}$ May 2019; accepted $3^{\text {rd }}$ Sep 2019)
}

\begin{abstract}
Ecological wisdom in traditional nomadic societies is formed in the process of production, life and spiritual belief. Nomadic settlements are multidimensional cultural heritage and cultural landscape types in post-modern society. This research takes Qiongkushitai village as the research object, and referring to the research methods and achievements of human geography and temporal geography, this paper discusses the spatiotemporal features and evolution of the traditional nomadic settlements under the process of rapid urbanisation. Taking local wisdom as the breakthrough point, two characteristics of the traditional nomadic spatiotemporal picture are condensed: (1) self-adaptability of nomadic spatiotemporal picture restricted by alpine resources, (2) multi-level nesting law of nomadic spatiotemporal picture. By extracting the typical fragments on the spatiotemporal axis, this paper attempts to propose a periodic activity model on the multi-level spatiotemporal scale in the nomadic space and time trajectory. This paper explores the evolution mechanism of traditional nomadic settlements driven by national policies, individual demands, technology, and market. The results of this study can provide information for the study of resilience construction in the construction of ecological civilisations in alpine pastoral areas in Western China.
\end{abstract}

Keywords: transhumance, adaptability of behavior, laws of nesting in multi-level space, ecological carrying capacity, conflict and acculturation

\section{Introduction}

Ecological wisdom is a positive part of tradition in life and production, which shows recessive and dominant characteristics. It shows the recessive temporal and spatial order in time, but in form, it reflects the physical form or lifestyle reflected by the skills and values of regional cultural genes (Hills, 2009). The production and life patterns of the Kazakh settlements living in the high mountains can provide basic living security on the premise that nomadic data can be provided, although other dynamic factors restrict the strength and depth of their inheritance or continuation. However, this kind of livelihood method, which is rooted in the natural pastoral environment, will also show the trend that the one aspect wanes, the other waxes in the grassland ecosystem chain.

Xinjiang alpine nomadic settlement, as one of the important carriers of grassland manland interaction, been concerned by the academic community and accumulated a wealth of academic achievements because of its special form. Through reviewing related research findings in recent years, reviewing, it mainly focuses on three aspects: (1) nomadic 
settlement and its related Noumenon Research: study on the Ecological View, Environmental behavior of Nomadic settlement; (Cui, 2002; Chen, 2017), Archaeological Observation of Prehistoric Nomadic Industry of the Western Tianshan Mountains in Xinjiang (Jia, 2018), Climate change and the local environment affected human activities in Heihe River basin (Shi et al., 2019), the characteristics of temporal and spatial, and its formation Mechanism (Meng, 2017), focus on recently implemented projects in the sequence of sedentarisation programs, the so-called Nomadic settlement (Ptackova, 2011); (2) Diversification of research perspectives: the study of the historical evolution of nomadic settlements on archaeological perspective, the tourism resources development such as tourism management and other nomadic settlements (Han, 2013; Li and $\mathrm{Yu}, 2019$ ), The production and lifestyle after settlement under cultural anthropology (Doumani et al., 2015), the change and evolution mechanism of national belief, etc. (Felr, 1994); (3) Study on Nested Scale of Nomadic Social Organizations: Grassland ecological restoration based on regional scale, regional mountain nomadic villages, etc. (Honeychurch, 2014; Chen, 2014), The Economy and Politics of Mountain Nomadism. Based on the above analysis, the research results of the nomadic spatio-temporal picture from the perspective of the ecological wisdoms are relatively few. The authors draw lessons from the research results of spatio-temporal behaviour of production and life in human geography and time geography. Taking Qiongkushitai village as an example, this paper makes a case study of Kazakh Alpine Nomadic Settlements in the Turks River Basin of Yili Prefecture and explores the ecological wisdom commonly found in traditional nomadic behaviour.

Based on a long-term field survey in the alpine pastoral areas of Yili Prefecture, Xinjiang, China, this paper explores the temporal and spatial characteristics of production, life and ecology of Kazakh herdsmen, who are the main body of traditional Alpine nomadic settlements. This paper attempts to explore the practical significance, cultural connotation, and the relationship between Kazakh traditional survival prospect and grassland ecology from the perspective of evolution mechanism driven by national policy, individual needs, technology, and market. The author hopes that other scholars will further explore the traditional nomadic society transformation period, herdsmen's modern living needs, and balance between human and natural ecology in pastoral areas, and provide some reference for the resilience building in the construction of ecological civilisations in pastoral areas in Western China.

\section{Materials and Methods}

In the process of rapid urbanisation, we should reflect on the practical value of ecological culture construction in traditional local wisdom. Each region is a local national badge (Crown, 2005). Ecological wisdom is one of the best symbols of regional cultural identification. Ecological wisdom is a stable structure and type inherited by a fixed group or region in the process of continuous reconstruction. It has the characteristics of self-discipline, local creation with the help of foreign knowledge, technology, formulation, and so on. The traditional Kazakh nomadic production, living in the exchange of material and energy with a grassland ecosystem, can achieve efficient coordination between resource utilisation and environmental conservation. Nomadic production is not only the result of adapting to nature but also the means of protecting nature to ensure the balanced utilisation of grassland and avoid the risk of natural disasters for herdsmen. The compensatory grassland ecological cycle mechanism, such as the roof-covering grassland model, compensation base ecological 
mechanism, moderate migration and nomadism, is rooted in the ecological view and environmental behaviour in nomadic activities, which condenses their ecological wisdom to live with the grassland (Chen, 2019). Ecological wisdom in traditional nomadic society is formed in the process of production, life and spiritual belief. It is also a multi-dimensional cultural heritage and landscape type for post-nomadic society. Therefore, the protection and development of cultural memory and cultural sites in post-nomadic settlements have important theoretical and practical significance.

\section{Constraint and adaptability of nomadic landscape}

The spatial and temporal characteristics of alpine grassland are important factors for restricting and encouraging nomads' access to resources (energy or information) and the way of using them regionally. Spatio-temporal differences are the response of human beings to the optimal use of time, which is believed to eventually lead to the growth of reproduction. In the new Darwinian theory, "adaptation"should be attributed to "moderate exchange" of energy (Torrens et al., 2010), information and technology or "Spatio-temporal threshold mechanism" (Meng, 2017). Time in the nomadic society is like an invisible bond, through the nomadic production and life of different time scales, through the visible and invisible space mapping and connected in the same system, namely, the nomadic grassland system. High-quality grassland resources appear seasonally in nomadic alpine settlements, so the survival strategy of effective utilisation of time is determined by the limited time of obtaining resources, and the time segment or seasonal length of each year. In the one-year periodic-cycle of nomadism, the core variables, such as the duration of effective utilisation of resources, the seasonal differences in the spatial and temporal distribution of available resources, the concentration and dispersion of resources, etc. The seasonal transition of nomadic production must be carefully planned and organised to reflect the adaptability to spacetime constraints.

\section{Spatio-temporal trajectory, space-time picture}

Spatio-temporal trajectory is the record sequence of the position and time of a moving object. As an important data type and information source (Yang, 2004; Gong et al., 2017) of spatio-temporal mapping, the spatio-temporal trajectory is the spatial appearance after different time overlaps in order in space. The spatial and temporal trajectory of grassland nomadism records Kazakh nomad stability, periodicity, regularity, the flexibility of nomadic behaviour, traffic logistics, and other information. The similarity and differences in characteristics of nomadic spatio-temporal trajectory data (nomadic time, linear space, behaviour node) were extracted under the typical space-time dimension, and the local ecological order in traditional nomadic behaviour was revealed. The recognition of typical regional characteristics was first reflected through a space and time picture.

The space and time picture is composed of recessive and explicit spatio-temporal order, behavioural pattern, materialised structure. The space-time picture is the sum of the material cultural landscape and the non-material cultural landscape formed under the same cultural or multi-cultural background. It is constructed by strong recognition symbols and is recognised and inherited. Local wisdom is transmitted to future generations through a concrete or visual form, and can also be regarded as the superposition and reproduction of spatio-temporal images through different historical periods. 


\section{Materials}

The typical Nomadic Settlements in the Turks River Basin of Yili Prefecture in Xinjiang, China (Figure 1), were selected as the research subjects. The research area is located in western Xinjiang, and there are a large number of traditional nomadic settlements in the high mountain areas. The region has features of typical landforms: undulating mountains, river streams with vertical and horizontal water sources, open and flat terrace grasslands, and other features to provide a natural place for nomadic activities. The Qiongkushitai village is located in southern Tianshan on the western part of the Tianshan Mountains. Two sides around the Wusun mountain, the two sides of the peak mountain stretch, adjacent barriers. The region has a long history of nomads, it is rich in cultural relics, less affected by the urbanisation process, and the traditional nomadic production behaviour remains relatively intact.

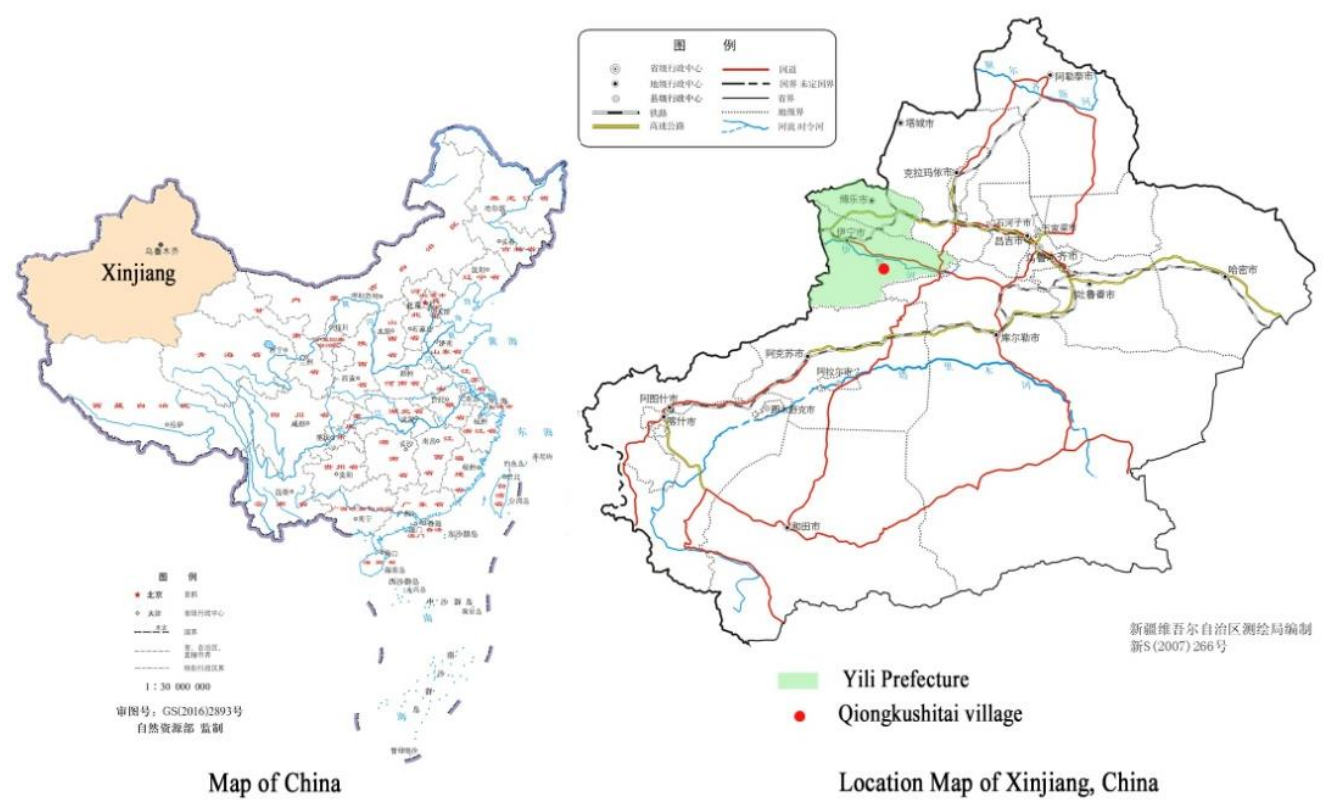

Figure 1. Sample Research Location Map . Drawn by author according to the official map of Xinjiang, China

\section{Historical evolution and general situation of Qiongkushitai Village}

(1) Location factors of nomadic settlements. The Qiongkushitai Village is located in the town of Karadara, Tex County, Yili Kazakh Autonomous Prefecture (locally known as "Five townships"), $90 \mathrm{~km}$ from the town government. It is a typical traditional pastoral village. The village's nomadic settlements are located within about $20 \mathrm{~km}$ of the Qiongkushitai River. Its territory is endowed with unique nomadic conditions: wide and flat terrain, warm climate, broad grassland, and suitable for farming and grazing.The village of Qiongkushitai has been a nomadic land for many ethnic groups since ancient times. Since the Western Han Dynasty, Wusun Ancient Road has been an important passageway connecting the north and south of Tianshan Mountains. A flat plateau between two streams provided a good place for business tours to stop here, hoard materials, and convert materials. It makes it possible to get together with the road. 
(2) Livelihood elements of nomadic settlements. The total area of the village is $136 \mathrm{~km}^{2}$, of which the total area of pastures is $84.7 \mathrm{~km}^{2}$, of which $18.0 \mathrm{~km}^{2}$ is winter pastures, 27.9 $\mathrm{km}^{2}$ is summer pastures, $31.6 \mathrm{~km}^{2}$ is spring pastures, and $7.2 \mathrm{~km}^{2}$ is clipping pastures. There is a total of 303 households with a total population of 1737. At present, animal husbandry and tourism are dominated by 967 people engaged in animal husbandry and 770 part-time workers in the tourism service industry, accounting for $44.3 \%$ of the total population. The development and utilisation of tourism resources in famous historical and cultural villages at the national level have promoted a major adjustment of the economic structure of traditional animal husbandry in this village. At the same time, the living standard of herdsmen has greatly improved, with the average annual income of herdsmen reaching 12,700 Yuan (Economic Income Statistics of Qionkushitai Village, 2017), which is currently higher than that of other pastoral villages in the same region. The development of tourism resources in pastoral areas has become a hot pursuit in the past five years. Traditional nomadic settlement resources have become scarce and precious. Local cultural heritages in the nomadic landscape of Qiongkushitai village, such as log settlements, inhabited grasslands, Wusun Pastoral Road, and the three revolutionary historical sites, reflect the evolution of nomadic cultural landscape and the results of local wisdom in the process of development (Figure 2).

\section{Research method}

The main methods used in this study are historical literature analysis, and settlement landscape theory and method. Based on the field survey data of alpine pastoral areas and data such as Xinjiang Urban-Rural Construction Yearbook, Yili Pastoral Area Industry Long-term Development Planning Platform, Xinjiang Pastoral Area UrbanRural Development Planning Implementation Guidelines. This study aimed at qualitative interviews with local nomads in a one-year nomadic cycle to obtain field data.The author analysed the space-time trajectory, internal characteristics, external expression and inheritance characteristics of nomadic cultural landscapes. The development of alpine nomadic settlements is a dynamic process. It is necessary to evaluate the regional characteristics and social structure in geography. The characteristics of nomadic cultural landscapes are identified and summarised by using ecological geography and economic research methods.

\section{Results}

\section{Typical characteristics of traditional nomadic space-time landscape}

The characteristics of nomadic production and life, such as social unit selforganisation, individual adaptability, time order, and nomadic spatial hierarchy, are the main factors that drive the dynamic space-time picture of nomadic life. Time factors, space factors, and social organisation factors determine the behaviour of the individual nomadic family as a unit and is restricted by nomadic ability (technical conditions, family labour force). The grass-roots organisation "Awule" provides the restriction effect and comprehensive guarantee of social authority, and optimises the combination of nomadic production activities of individual families and the conversion behaviour under the restriction of grassland resources under different seasonal elevation gradients. In the space-time, a social organisation unit shows a multi-layer nested human landscape picture. 


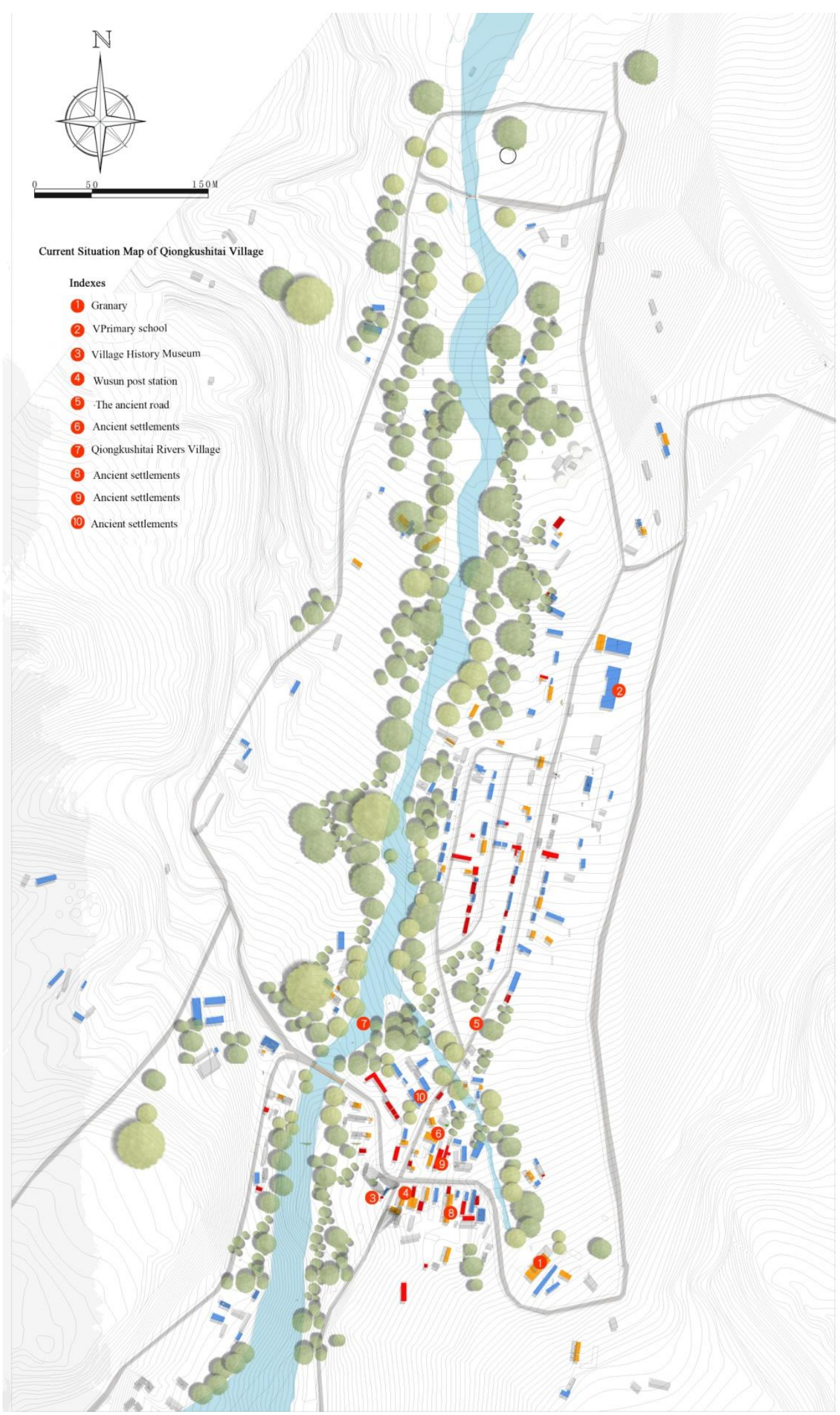

Figure 2. Current Situation Map of Qiongkushitai Village. Self-drawn by author 


\section{Adaptability of nomadic space-time picture under the restriction of resources}

Mixed model of migration and fixed residence in nomadic space-time scene. The traditional nomadic settlement form is the minimum spatial scale and unit for nomads to engage in group production and life, and it is also the iconic element of nomadic space-time picture. The pasture and settlements show two spatial patterns of aggregation and dispersion in the nomadic complex space. The other is that pastures and residential space are very close. The main factors of the seasonal and elevation differences in the distribution of grassland resources, determine the aggregation and fragmentation of the spatial form of settlements. In the nomadic cycle of one year, the living space has obvious vertical zonality. The spatial characteristics of settlements reflect the interaction between human activities and the natural environment in the process of nomadic migration from the micro-scale. The living characteristics of QiongkuShitai Village are typical in the alpine pastoral areas of northern Xinjiang. The second-order flat area of the valley between the Wusun Ancient Road and the Qiongkushitai River is the first choice for the settlement. Its overall form is banded, local concentration, the courtyard is patchy, the building along the river and contour parallel to the horizontal expansion, but also perpendicular to the longitudinal continuation of the contour. The scattered distribution of residential groups corresponds with the characteristics of nomadic production.

Mobile residence under the adaptation of livelihood resources. In the process of periodic nomadic space-time picture, there are complete identifiable or identifiable nodes in nomadic adjacent time-space, and the residential space changes with the behaviour of the nomadic stage, which is the most typical. For example, when spring and autumn pastures migrate to winter pastures, the form of residence changes from "Haita" (temporary portable type of residential space) to "winter nest" (winter residence), and summer pastures-assembled felt houses for spring and autumn pastures. Portable features are suitable for long-distance transitional residences. Based on the period and frequency of building use, construction type and the degree of expenditure, residential forms can be divided into permanent type, stage type, and transition type.

The first choice of settlement location and the hierarchical selection mode of secondary dependency. Based on the analysis of the settlement layout and the shape of Qiongkushitai village, the relationship between settlement, grassland, and water source, the hierarchical selection model of nomadic settlement environment selection was put forward, which exists the first choice and the secondary dependency. The preferred land is usually flat valley, leeward, and riverside for water needs, especially in spring and autumn pastures, the attachment of water resources is stronger than summer pastures. Because of the high spatial distribution density of water resources in summer pastures and the short rainfall cycle in mountain areas, settlements are not necessarily built near the river, and usually have convenient commuting distance, which is also closely related to the quality of grassland resources.

\section{Linear spatio-temporal presentation of nomadic spatio-temporal trajectory: transition track and pastoral space}

Periodic transition is one of the core elements of the nomadic landscape, and it is also one of the symbols that nomadic space-time picture is strongly recognised. The track of nomadic space is jointly determined by three factors: the specific logic of the 
spatial and temporal allocation of nomadic subjects and resources, the coherent time order, and the flexible nomadic behaviour.

(1) Stable and dynamic characteristics of nomadic spatio-temporal trajectory. The transitional pastoral path is the product of the nomadic spatio-temporal track. The migratory transition behaviour and the individual family each have the zonation and verticality of the spatial distribution of grassland resources, which determines the dynamics and stability of the nomadic spatio-temporal trajectory in which the nomadic herdsmen complete the periodic migration. Dynamics refers to a local strategy for nomads to deal with natural disasters in a short period of time. Stability refers to the maintenance of long-term effectiveness of high-quality grassland resources and the long-term stability of the selected and optimised linear space. The village of Qiongkushitai belongs to the alpine transition settlement, and the herdsmen have a certain time, order and route, so they have formed a transitional pastoral road with spatio-temporal order (Huang, 2013). Qiongkushitai pastoral road and the linear spatial scale is $110 \mathrm{~km} \leq 150 \mathrm{~km}$. The characteristics of long transition route and strong stability; River valley meadow, Zhongshan grassland and alpine grassland are distributed at an average altitude of $1020 \mathrm{~m} \leq 2680 \mathrm{~m}$. The long-span characteristics of grassland resources at elevation determine the dynamics of nomadic trajectory (Figure $3)$.

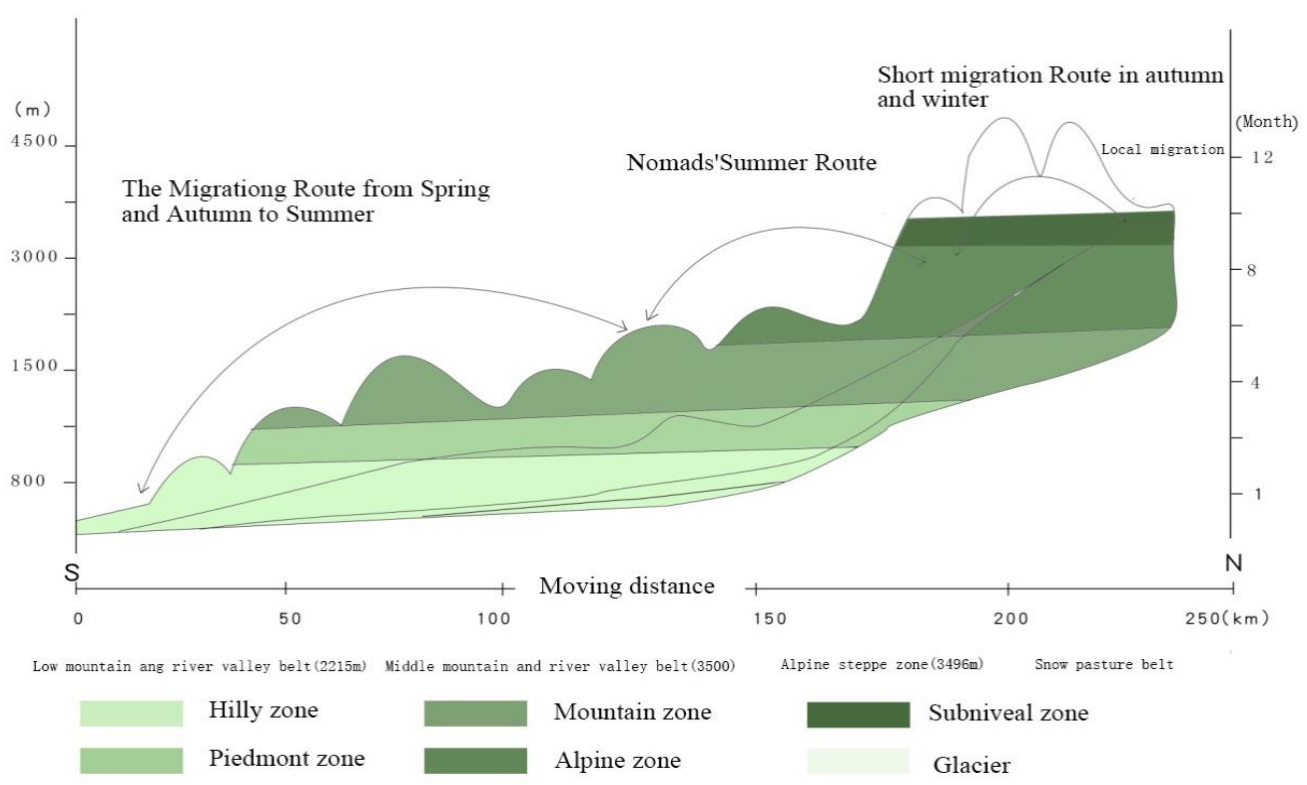

Figure 3. The temporal and spatial trajectories of migration in the one year period of Nomadic settlement. Author redraws according to the illustration of "Transition and Modernity of Nomadic Society: Mountain Volume" (Center for Rural Environmentsocial studies, 2015)

(2) Long-term "life path" and short-term "daily path" of nomadic spatio-temporal track. The construction of a nomadic path over long period is the representation of nomadic ecological strategy in time-space coordinates. The long-term migration strategy of alpine nomads has opened up a long-term "Life path" for their seasonal migration to summer pastures. It embodies the ecological values of the sustainable development of nomadic behaviour and ensures the possibility of the existence of nomadic picture for a long time. 
To a large extent, nomadic individuals can flexibly adjust their nomadic behaviour and make strategic decisions on the basis of following their natural laws, which is specifically reflected in the dynamic characteristics of the "daily path"in a short period of time. The nomadic "daily path" with one year as the cycle, constitutes a long period of "life path" of nomadic production in alpine grasslands. The short period of "daily path" is the basic element of the inheritance of grassland production mode, which ensures the continuation of the long period of "life path". The short-term "daily path" promotes the existence of the long-term "life path"of nomadic activities. Each type of nomadic behaviour can find its place on the timeline of the "daily path" for a short period of time, and the nomadic activity per unit time presents a typical spatial or behavioural reflection on the "fragment order" (Table 1). The short-term nomadic track depicts the seasonal movement trends of nomads in the daily periods, such as the change of space place in spring, summer, autumn, and winter, the change of living space, and daily nomadic behaviour such as lambing, fattening, grabbing, captivity, stocking.

Table 1. Space-time order of "daily track" in a short period. Self drawn by author

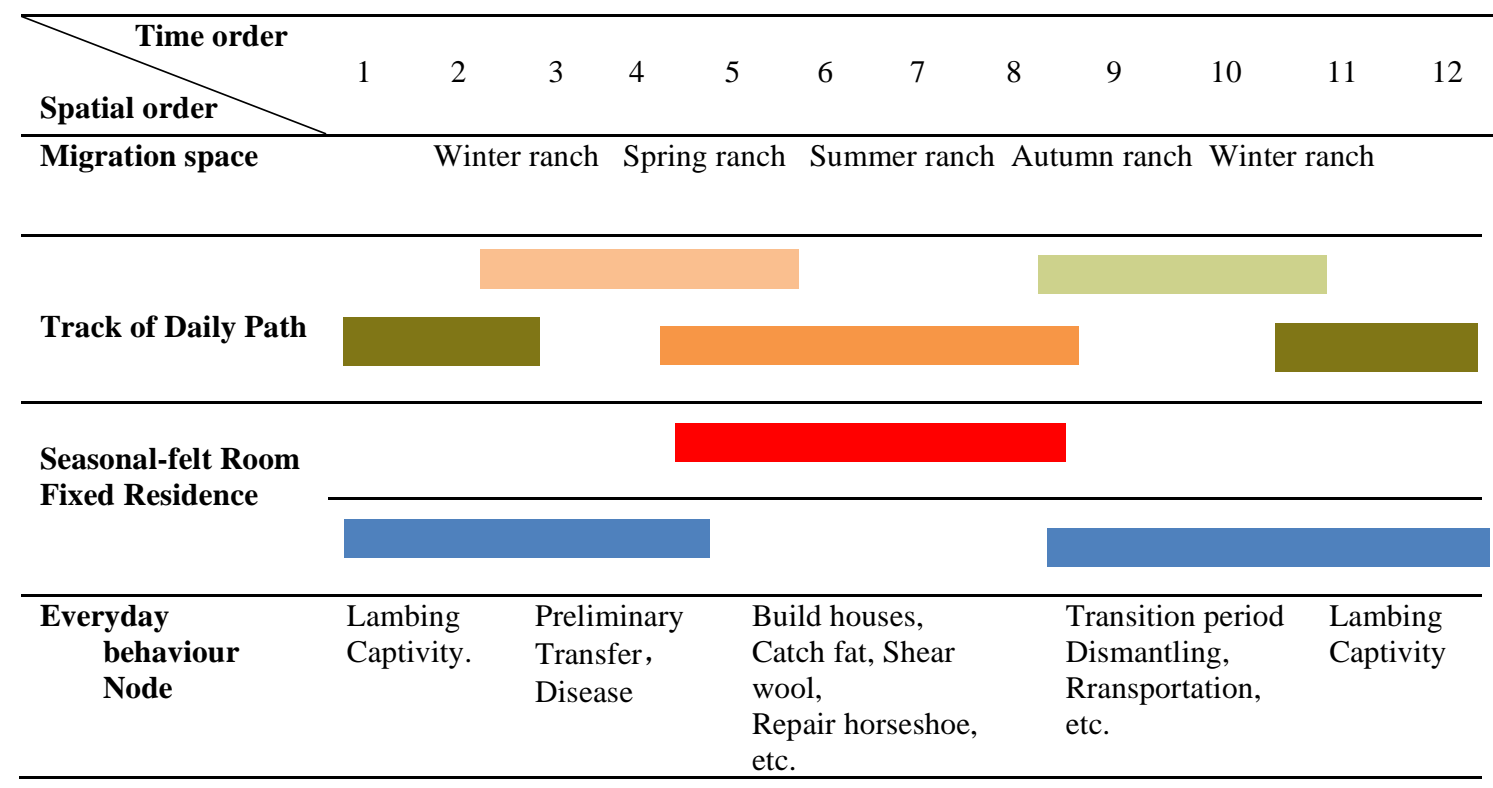

\section{Multi-level nesting law of nomadic space-time picture}

Nomadic production and life are subject to regional resource conditions (the types of grassland resources and their advantages and disadvantages are distributed in time and space). The self-characteristics of nomadic production and life (the family is the unit + the group mutual aid mode of work) and other factors determine that the nomadic space-time picture shows the law of nesting at different levels. It is embodied in the nesting of the local order of the social unit and the nesting of the multi-level "centre-periphery" on the spatio-temporal scale of the relationship between man and land.

Local order nesting of social units: the organic combination of individual family and "awule" organization. "Local order nesting of activities" emphasises the relationship between human activities and the allocation of space-time resources needed for their 
occurrence, implying the temporal arrangement and spatial combination of the resources needed behind human behaviour. Drawing lessons from its achievements, the interpretation of the grass-roots organisational order of nomadic society is helpful to explore the wisdom of building resilience in the construction of nomadic ecological civilisations. The grass-roots organisation of "Awule" is the builder of the grass-roots order in the traditional nomadic society. The stability of grass-roots social organisations depends on the ownership of the means of production, so each basic nomadic unit has its own territory, tribes have their own transfer routes, and customary law has a strong binding effect on life and production, to ensure the order of social organisations on a large scale.

The relationship between the nomadic family unit and "Awule" organisational behaviour is often inclusive and restricted. A series of activities of nomadic families aim at achieving individual goals: survival and nomadic activities are necessary to maintain survival on an individual scale. The goal of "Awule" organisational behaviour to maintain the basic order of nomadic society is to continue the fundamental activities of the existence and inheritance of such organisations and solidified into a symbolic organisational model. Nomadic individuals and organisations depend on each other and are reflected in the combination of nomadic behaviours. The nomadic space-time picture carries the activities of family, society, and nature. The temporal and spatial allocation of grassland resources and space is relatively regular in nomadic behaviour. It profoundly depicts the ecological process of the utilisation of regional resources by nomadic subjects under the influence of internal order.

Multi-level "center-sub-core-boundary" nested model of man-earth relationship on the spatio-temporal scale. The traditional settlement is not only the material support of human survival, but also the symbol of living space. The location of settlement integrates the basic elements of human survival mode and environmental choice (Han, 2008). Under the alpine nomadic production and lifestyle, the settlement environment follows the upward order of the vertical space of alpine grassland,medium alpine grassland, valley grassland, secondary grassland, and the diffusion law of successive circles in horizontal space. Nested model shows the relationship between human and land in Alpine nomadic ecological environment. This paper summarises the basic model of traditional man-land relationship in nomadic alpine settlements: horizontal multi-layer spatial nesting, and vertical upward "centre-sub-core-boundary" nesting model with grass migration. Through the settlement environment selection of Qiongkushitai village, the human-land relationship of nomads in the small watershed of Qiongkushitai River is re-examined. The multi-layer spatio-temporal nesting model of nomadic circle is a dynamic model which is used to deal with the complex nomadic ecosystem in balance and sustainability. For example, the location relationship between a family's multiple places of residence and grassland determines the man-land relationship model of settlement location.

\section{Discussion}

\section{Conflict and Integration: the evolution of traditional nomadic space-time picture}

The nomadic space-time picture is rooted in the space-time accumulation of specific natural conditions and human activities. It is reproduced through a series of time and space chain continuity such as a series of forms and activities in a specific alpine grassland space. Nomadic resources determine the type of production and mode of 
production, while the nomadic mode of production determines the way of life and social organisation of nomads; lifestyle and social organisation form condense into grassland culture and art. The spatial layout of traditional nomadic production and life and the timing arrangement of production and life are deeply affected by industrial economic structure, family type, and technological innovation. When the traditional material space disappears (or the function is replaced), the nomadic picture may be presented in another way: the appearance of exhibition, fragmentation, and symbolization, then the grassland nomadic picture loses the vitality of expression and the path of inheritance.

\section{The drive of national policy of settlement and pastoral settlement}

Spatial compression and linear fracture of traditional nomadic spatio-temporal trajectory.

Functional displacement of the pastoral resources. "For nomadic animal husbandry, the key factor in its development lies not in possession of grassland, but more in the right of migration, only in the process of migration with the sun" (Han, 2003). Stable and continuous transition is the most essential feature of nomadic culture. When grassland resources are replaced by conversion paths are separated, conflicts between pastoral roads and modern roads overlap, nomadic production becomes settled captivity. As a result, the seasonal and spatial continuity of grassland nomadic spacetime track is broken, the nomadic chain is fragmented, and the traditional nomadic picture shows fragmentation. The spatial evolution and utilisation pursuit of the production behaviour of the micro-subject is the main internal driving force for the emergence of the above situation.

Under the background of rapid urbanization, the traditional nomadic space is compressed, the nomadic trajectory is discontinuous. There are problems in the process of redistribution and use of grassland resources. Breaking the traditional model of resource allocation, the traditional model of life and production has been gradually dispelled, and the living form has moved to a fixed-point residence with the adjustment of production structure. The grassland boundary is fenced, the grassland resource type is single, the grassland space radius becomes smaller, and the controllable transition path is shortened. The people and cycles who undertake the transition are compressed (domestic work is replaced by employment, mechanised transfer, shortening the transition period), the surplus labour force begins to shift to other livelihoods, and grassland resources no longer carry the current multi-level demand. Captive cultivation, the replacement of resources in different places, and animal husbandry production have broken the barriers to the distribution of spatial resources, cross-regional grassland artificial planting and anti-ecological farmland grass which is one of the ways to solve forage resources.

\section{Individual needs drive the elimination of local intelligence system in traditional pastoral society}

The settlement of nomads is a profound process of social and cultural change. The settlement of nomads is a process of re-socialisation which differs greatly from their traditional social culture. Nomads will face, and inevitably experience, the problem of re-socialisation in the process of settlement. The traditional form of "Awule" and "family unit co-production", which exists in nomadic social organisations, has been replaced by "individual family type, modern employment production". Standardisation 
and accuracy of time cost. The mechanised, long-distance, cross-regional fragmented transfer behaviour model promotes the local wisdom spread in the traditional transition field to be concentrated and dispelled, and the compression of time and space promotes the acceleration of the rhythm of time and the overcoming of the hindrance of time to space caused by it. The temporal and spatial constraints and incentives of nomadic daily life trajectory have been reduced. In order to better control the time cost, animal husbandry production promotes the standardisation of time cost: the quantification and accuracy of time in the nomadic process, but with the change of phenology, the time cost is controlled within a certain range. The precision of the cost of production and living commute is reflected in the evolution of residence: from a nomadic lifestyle to settlement, felt house, semi-settlement wooden house, settlement log building, mobile residence mode and semi-settlement residence that changes with the season, the ownership of grassland changes, evolution from mobile and semi-settled to permanent residence. In order to meet the current needs of life, herdsmen build so-called high-end living environments, but it destroys the balance of the grassland water system, vegetation, and biology. The grasslands with slopes were filled with raw soil foundations, and modern buildings were built on them. These buildings are incompatible with the original grassland environment (Figure 4).
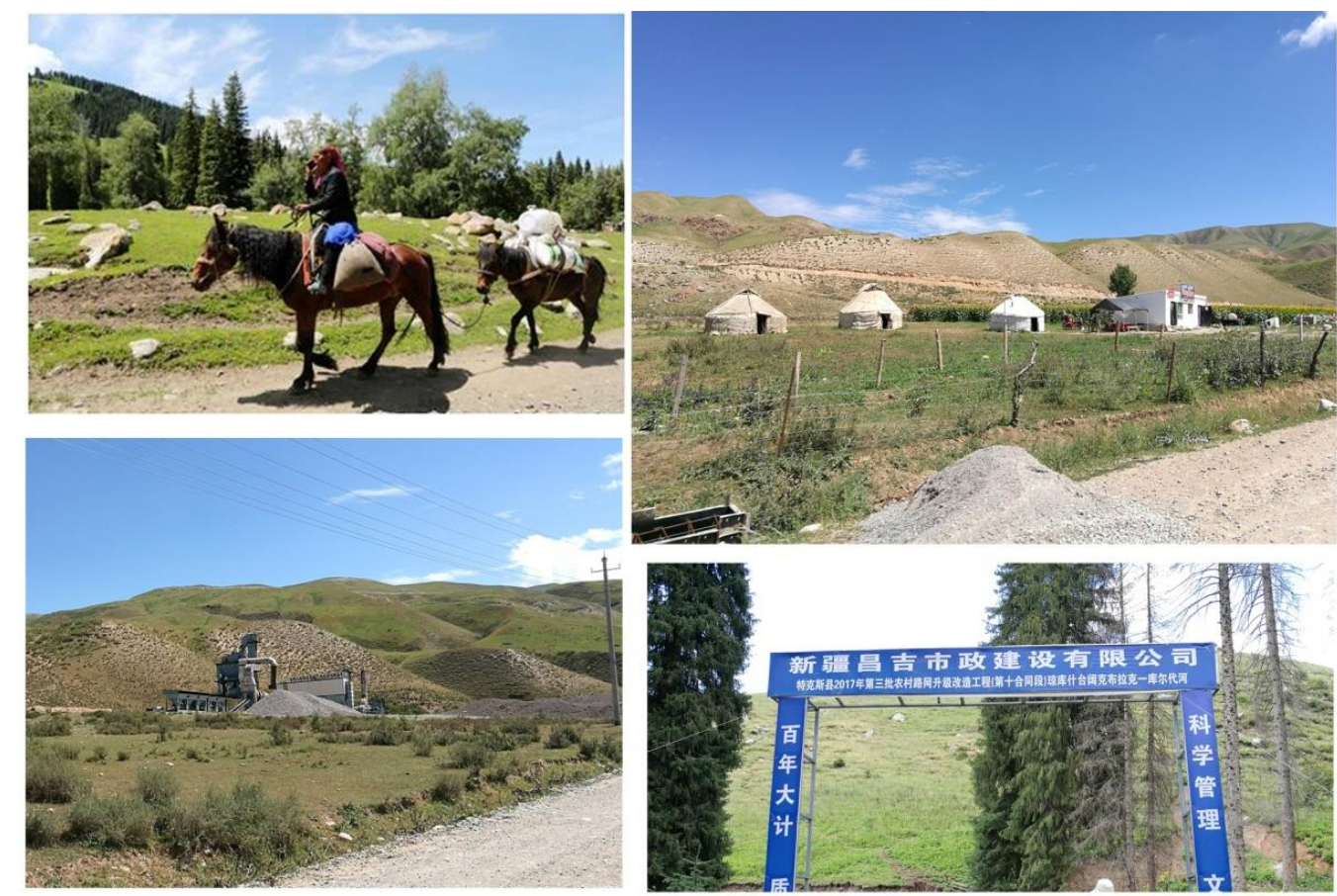

Figure 4. Driven by information, technology, and materials, it is possible to expand the living space. Photographed by author

Qiongkushitai's settlement began construction in 2012. It was originally a traditional settlement and reserved feed land in the spring and winter seasons of the pastoral village. The scale of the settlement is larger than that in other areas. Apart from the first and second group of displaced herdsmen, some land (originally pasture) for other later settled herdsmen has been reserved. There are 87 settled herdsmen. The land composition of settlements is composed of public grassland and pasture of 
herdsmen. In order to apply for settlements, the herdsmen need to take out three acre of land use rights to solve the land problem occupied by settlements. The settlement is facing the river, has convenient transportation, relatively open terrain, and good natural scenery. The water construction of the settlement is one of the projects of combining agriculture and animal husbandry, and developing pastoralists by settling down. The government hopes to develop tourism resources to solve the livelihood problems of some pastoralists after settling down. At present, it has attracted local herdsmen and some people from surrounding counties to settle and run tourism. In early 2017, the settler, Karze, transferred 56 acres of summer pasture and 15 acres of reserve pasture from his home. There is a steady income every year. At the same time, a Herdsman's Folk Tourist Inn has been built in the original settlements (not planned into the new settlements). Kalze has three children; two younger sons are in high school in Turks County. He and his daughter usually run their own camps together, and they are relatively wealthy. In the new settlement, Kalze has its own housing (herdsmen put out 60,000 yuan of construction funds, local government provides planning and other supporting facilities for the construction). When applying for settlement, the property rights of the housing belong to herdsmen, but no real estate certificate has been issued yet. For example, to protect herdsmen and social stability, settlements cannot be rented and sold out in principle, but there will be individual herdsmen to rent settlements to other people. The settled herdsmen have adapted to the present life, and the family economic structure has increased the additional income of the tertiary industry (pastoral transportation, tourism services, special breeding.) from the original pure income of animal husbandry production.

As a typical case, Qiongkushitai village is demonstrated in this area. Under the advocacy of governments at all levels, nomadic villages with tourism resources and convenient transportation conditions have begun a new round of "symbolic economy" rising, which repeats the upsurge of the urban symbolic economy. As famous villages and towns of history and culture, and as the main body of official protection law, their names will attract a large number of tourists. With the improvement of other factors, nomadic cultural resources, as an economic resource, enhance the income of local residents and also become a sign of local cultural tourism. The rise of symbolic economy is due to two reasons: one is the economic decline within the city relative to the suburbs. Cultural symbols play an important role in the process of the revival of the urban centre area. The other reason is the expansion of the investment field, and culture has become a new investment field. It has become a scarce resource for internal demand and external pursuit of dividends. Through the investigation of production and life in Qiongkushitai Village, it is concluded that the mode of production has changed after settlement.The traditional local wisdom heritage, agglomeration and decomposition, and the new life and production mode have become popular in pastoral areas (Figure 5).

\section{Conflict and fusion: dynamic adjustment of nomadic picture induced by new technology}

Technological change is a traditional factor of production for the sustainable and rapid development of settlements. Through the author's field investigation in the Tex River Basin, it is found that the application and popularisation of the new technology in the traditional pastoral areas is the main driving factor for the renewal or integration 
of the traditional nomadic space-time picture, and it is also the inevitable result of social development. Extract $t$ Three types of typical case descriptions:

(1) The innovation of production tools. The changes of transit vehicles: traditional horses, camels, flat cars vs horses and motor vehicles; innovation of weeding tools: traditional long handle sickle vs weeding mechanisation;

(2) Innovations of living facilities. The change of communication tools: horseback riding to transmit information vs modern mobile communication tools. The location of water resources and access to the reduced constraints: water-by-water living vs now long-distance across the space-time construction of water facilities. Comprehensive strategies to deal with disasters: local wisdom word of mouth vs rapid emergency system, mechanised long-distance commuting support.
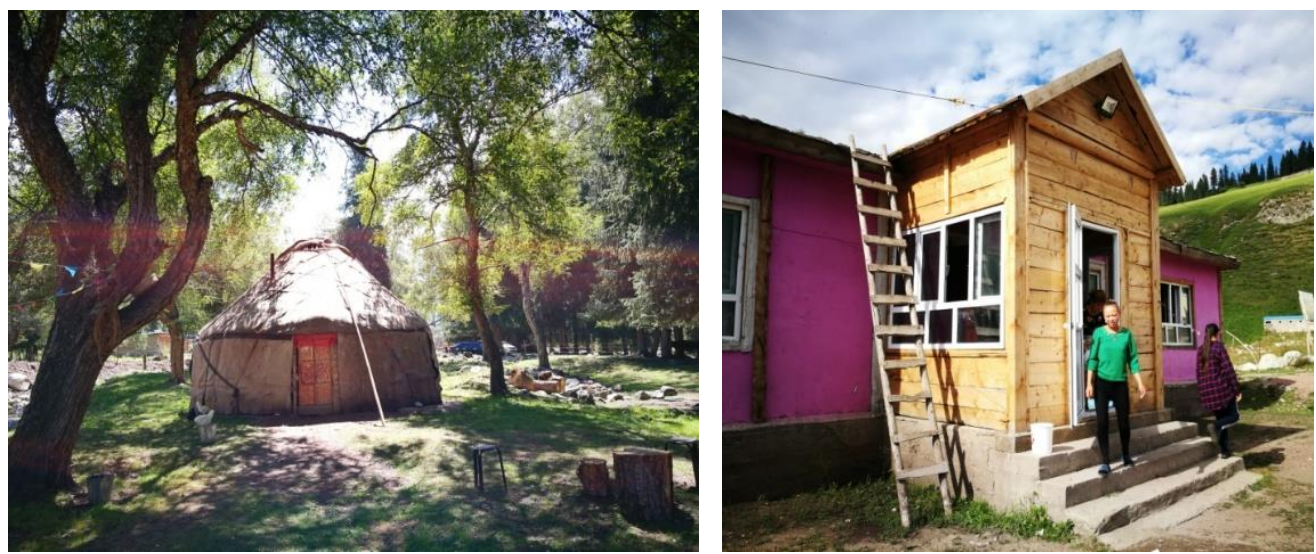

Figure 5. Forms of Residence before and after Settlement. Photographed by author

(3) Technology and Market Drive. Adjustment of nomadic landscape in the postindustrial era dynamic adjustment of nomadic space-time picture (cultural landscape). When the frequency of new technological innovation is accelerated, and the popularisation of application is high, the process of material production reflects the compression of space-time picture and the rapid change of spatio-temporal feeling. For example, the multi-level coverage of vehicles and roads has made it possible to explore potential sites for the spatial expansion of nomadic production. At that time, the boundary of space was broken through, and the cost of time was reduced to the lowest in geography and space. The acceleration of capital flow and exchange speed reconstructed the spatial picture of nomadic production and consumption. Postmodernist grassland nomadic cultural landscape, time and space experience is the result of the combination of various social resources. The traditional nomadic time and space picture has three possibilities: collage, reconstruction, and disintegration. Driven by external and internal forces, it has completely broken the rank, stability, and continuity of traditional time and nomadic space, and only the fragmentation of sporadic temporal and spatial characteristics has been retained and reconstructed in a single way. The segmented nomadic space-time picture is used as a display label and developed as a selling point (Harvey, 2003). The cultural tourism brand promotes the experience of this nomadic cultural landscape of a pastoral village by saying: return to the most primitive nomadic settlement: experience nomadic slow life here. It is not a real experience of grassland nomadic time and space, but an illusory experience 
created by the collusion of capital and media to satisfy the strange experiences of tourists. We should understand the unique cultural landscape characteristics contained in different agricultural and cultural heritage and provide method support for the rational utilisation and protection of important agricultural, and cultural heritage in our country (Hu et al., 2018).

\section{Conclusion}

Based on the case study of Nomadic Settlements in the Turks River Basin, this paper puts forward two characteristics in the space-time landscape of nomadic alpine settlements: (1) the adaptability of nomadic space and time landscapes under the constraints of alpine grassland resources, and (2) multi-level nesting rule of nomadic space-time landscape. Nomads are passively adapted to regional resources in the process of nomadic production and life. However, the nomadic subjects dynamically adjust their nomadic behaviour according to their own conditions (population, technology, grassland resources), and form a multi-layer organic combination of mutual social organisations, periodic stability of nomadic space and time landscape, living scale and multi-point migration of space.

Driven by national policy, individual demand, technology, and market guidance, nomadic settlements in the process of urbanisation are out of order in production and life of traditional nomadic society, and their ecological carrying capacity is weakened due to the combined effects of external and internal forces such as policy implementation, modern technology, and market guidance. The short-term "daily path" of nomadic production and life characteristics will be decomposed or split, the longterm "life path" will disappear or be replaced by another form, and the "modern" nomadic landscape will be reconstructed. The reconstructed nomadic landscape is embodied in two aspects: (1) nomadic economy under the change of economic structure is no longer the core of family income. Herdsmen need to manage other economic types on the basis of traditional nomadic production to meet the needs of grassland resources, ecological deterioration, and improvement of living standards. For example, they are engaged in settled agricultural production, tourism, and nonagricultural production in cities, and (2) technology and market incentives, traditional natural nomadism to fixed-point non-organic pasture change. The practice of fixedpoint industrialised captive farming production mode in traditional alpine pastoral areas has reduced the frequency and distance of transfer, weakened the constraints of grassland space-time resource allocation, weakened the original intimate relationship between nomads and livestock, water and grass, and gradually diluted or replaced the traditional alpine nomadic culture.

This article serves as a starting point for further research concerning the development and change of lifestyle for the nomads after settlement life and fixed grazing production. We should select samples with universality to ensure the validity of the universality of the research results. Due to the highly dispersed spatial pattern of nomadic alpine settlements and the constraints of researchers' energy and academic levels, it is hoped that comparative studies can be carried out in the latter part of the study to select sufficient samples within the geographical units of small watersheds to make up for the deficiencies in this study. Suggestions for future research would be to pay attention to the integration and adjustment of the old and new nomadic scenery in nomadic alpine settlements, retain the essence of the traditional, nomadic, and 
humanistic landscape or the reconstruction of the new cultural landscape of the main components to meet the needs of the new era. The assessment of regional ecological environment carrying capacity of nomadic settlements should be paid attention to by scholars, and the research on the coordinated development of nomadic livelihood needs and ecological protection should be deepened to explore the theoretical and practical path suitable for the protection and development of traditional nomadic settlements.

Acknowledgements. This study benefited from the opinions of the reviewers and editors of the journal, and was supported by the National Social Science Foundation of China: "Study on Landscape Protection of Oasis Historic and Cultural Villages and Towns" (14G126) and "Study on the Cooperative Mechanism of Water Cultural Heritage Protection and Rural Revitalization in Xinjiang Section of the Silk Road" (19XMZ046).

\section{REFERENCES}

[1] Center for Rural Environmentsocial studies (2015): Transition and Modernity of Nomadic Society: Mountain Volume. - China Social Science Press, Bejing.

[2] Chen, X. J. (2014): Response and influence of grassland property rights change in Kazakh pastoral society - Taking Fuyun County of Altay, Xinjiang. - Journal of Xinjiang University (Philosophy, Humanities and Social Sciences Edition) 1: 62-67.

[3] Chen, X. J. (2017): Altai Mountain Nomads-Ecological Environment and Local Knowledge. - Social Science Literature Press, Beijing.

[4] Chen, X. J. (2019): Lost grassland sacredness: perspective of grassland ecology crisis. Journal of Culture Vertical and Horizontal 2: 128-135.

[5] Crown, M. (2005): Cultural Geography. - Nanjing University Press, Nanjing.

[6] Cui, Y. (2002): Resocialization of nomadic settlements. - Journal of Xinjiang Normal University (Philosophy and Social Sciences Edition) 4: 76-82.

[7] Doumani, P. N., Frachetti, M. D., Beardmore, R., Schmaus, T. M., Spengler, R. N., Maryashev, A. N. (2015): Burial ritual, agriculture, and craft production among Bronze Age pastoralists at Tasbas. - Journal of Archaeological Research in Asia 2: 17-32.

[8] Felr, S. (1994): Drought, Nomadism, Grassland. - China Construction Industry Press, Beijing.

[9] Gong, X., Tao, P., Jia, S., Ming, L. (2011): Progress in spatiotemporal trajectory clustering. - Journal of Progress in Geographic Science 30(05): 522-528.

[10] Han, M. (2003): On the interaction between animal husbandry production and environment in northern China. - Journal of Geographical Research 1: 89-95.

[11] Han, M. (2008): Settlement Environment Selection and Human-Earth Relations in the West Liaohe River Basin since Holocene. - Journal of Geographic Studies 5: 118.

[12] Han, M. (2013): On the southward moving of agricultural technology and the economic center of ancient China by northern migrants. - Journal of Study of Chinese History 4: 117.

[13] Harvey, D. (2003): Study of the Origin of Cultural Change in the Post-modern Situation. - Beijing Commercial Press, Bejing.

[14] Hills, E. (2009): On Tradition. - Shanghai People's Press, Shanghai.

[15] Honeychurch, W. J. (2014): Alternative complexities: the archaeology of pastoral nomadic states. - Journal of Archaeological Research 4: 277-326.

[16] Hu, Z., Qingwen, M., Peilin, L. (2018): Exploration of Cultural Landscape Characteristics Recognition of Agricultural Cultural heritage. - Journal of Economic Geography 38(02): 187. 
[17] Huang, S. Y. (2013): Pastoral inspection in alpine transition style: taking Turks County as an example. - Journal of Yili Normal University (Social Science Edition) 33(02): 27 35 .

[18] Jia, W. M. (2018): Archaeological Observation of Prehistoric Nomadic Life: Analysis of Prehistoric Settlements in the Western Tian shan Mountains of Xinjiang. - Journal of Study of Western Regions 03: 63-75.

[19] Li, J., Yu, J. H. (2019): The change and adjustment of traditional culture of nomadic ethnic groups: a case study of Nadamu, Henan County, Qinghai Province. - Journal of Qinghai Social Sciences 2: 180-185.

[20] Meng, F. L. (2017): Study on spatial characteristics, types and causes of formation of historical and cultural villages and towns of oasis type in Xinjiang. - Guizhou Ethnic Studies 38(01): 95.

[21] Ptackova, J. (2011): Sedentarisation of Tibetan nomads in China: Implementation of the nomadic settlement project in the Tibetan Amdo area; Qinghai and Sichuan Provinces. Journal of Pastoralism 9: 68-77.

[22] Shi, Z., Chen, T. T., Storozum, M. J., Liu, F. W. (2019): Environmental and social factors influencing the spatiotemporal variation of archaeological sites during the historical period in the Heihe River basin, northwest China. - Journal of Quaternary International 25: 34-42.

[23] Torrens, R., Hong, C., Yan, P. (2010): Time budget and hunting and collection techniques. - Journal of Southern Heritage 1: 147-153.

[24] Yang, N. (2004): Prospects for local knowledge, local sense and cross-regional research. - Journal of Tianjin Social Science 6: 119-125. 